\title{
An Enhanced Energy Aware Coverage Preservation with Static Clustering for WSN
}

\author{
Arti Rana \\ M.Tech CSE \\ Uttarakhand Technical \\ University \\ Dehradun, India
}

\author{
Anshika Garg \\ Assistant Professor \\ Uttarakhand Technical \\ University \\ Dehradun, India
}

\author{
Sandip Kumar Chaurasiya \\ Assistant Professor \\ Dept. of Comp. Science\& Engg. \\ Dit University \\ Dehradun, India
}

\begin{abstract}
A wireless sensor network consists of a huge number of sensor nodes associated via wireless medium. Nodes in a WSN are organized with limited battery energy and thus improvement on network lifetime by reducing energy-usage is of extreme important. One of the solutions to minimize such energy-usage is associate clustering of network nodes. In this paper an associate clustering scheme in WSN is proposed where cluster heads are formed by creating a probable associate list is in descending order in which cluster heads are organized according to their residual energy. We have conducted simulation-based evaluations to compare the performance of EEACPSC against Energy Aware Coverage Preservation and Lifetime Enhancement Protocol (EACPLEP) and Enhanced-Energy Efficient Protocol with Static Clustering (E3PSC). Our experiment results show that EEACPSC outperforms EACPELP and E3PSC in terms of network lifetime and power consumption minimization. And its results come out in 3D form.
\end{abstract}

\section{Keywords}

Cluster head, Network Lifetime, Energy Efficiency, Wireless Sensor Network.

\section{INTRODUCTION}

Innovations in industrial, home and automation in transportation represent smart environments. Data for smart environments are obtained through Wireless Sensor Networks (WSN), where thousands of sensors are deployed at different locations operating in different modes [1]. A sensor network is capable of sensing, processing and communicating which helps the base station or command node to observe and react according to the condition in a particular environment (physical, battle field, biological) [2]. Sensor network protocols have a unique self-organizing capability. Another interesting feature of WSNs is that the sensor nodes cooperate with each other. Sensor nodes have an in-built processor, using which raw data are processed before transmission. These features facilitate wide range of applications of WSNs ranging from biomedical, environmental, military, event detection and vehicular telematics [3].

In this paper we assume a sensor network model, similar to those used in [4]-[6], with the following properties:

- All sensor nodes are immobile and homogeneous with a limited stored energy.

- The nodes are equipped with power control capabilities to vary their transmitted power.

- None of the nodes know their location in the network.

- Each node senses the environment at a fixed rate and always has data to send to the base station.

- Base station is fixed and not located between sensor nodes.
In this paper, we propose EEACPSC (An Enhanced Energy Aware Coverage Preservation with Static Clustering), a hierarchical static clustering based protocol, which eliminates the overhead of dynamic clustering and as a result prolongs the network lifetime. In this scheme, cluster heads are selected by creating a probable associate list where cluster heads are organized according to their residual energy and all the rounds are divided into iteration. The rest of paper is organized as follows. Section 2 describes the proposed scheme followed by the supporting algorithm. Performance-evaluation and simulation results are presented in section 3 . The entire work is concluded in section 4 .

\section{PROPOSED SCHEME}

This section contains the proposed scheme, An Enhanced Energy Aware Coverage Preservation with Static Clustering (EEACPSC) followed by the algorithm of the proposed scheme. The present scheme is a modification of an existing scheme, An Enhanced Energy-Efficient protocol with Static Clustering(E3PSC)[7] and Energy Aware Coverage Preservation and Lifetime Enhancement Protocol(EACPLEP) [8]. In the present work, cluster heads are choose by creating a probable associate list for next $\mathrm{n}$ number of rounds and the entire cluster heads are organized according to their residual energy.

\footnotetext{
2.1 Algorithm (EEACPSC)

1.BEGIN

/* Setup Phase (tasks are performed by the base station) $* 1$

2.Form $\boldsymbol{k}$ clusters taking $\mathrm{n}$ randomly distributed nodes based on distance

/* Same as EACPLEP*/

3. For $\mathrm{i} \leftarrow 1$ to $\mathrm{k}$

4. Compute mean position of node distribution,

Pmean in $_{\text {cluster }}$

5. for $\mathrm{j} \leftarrow 1$ to $\mathrm{m}_{\mathrm{i}}$

$/ * \mathrm{~m}_{\mathrm{i}} \rightarrow$ No. of nodes in cluster $_{\mathrm{i}} * /$

6. Compute distance of $\boldsymbol{n o d e}_{j}$ from $P_{\text {mean i }}, \boldsymbol{d}_{\text {mean } \boldsymbol{i}}$

7. End For

8. Select Temporary Cluster Head $\left(\mathrm{TCH}_{\mathrm{i}}\right)$ randomly for cluster $_{i}$

9. Create TDMA schedule for the nodes of cluster $_{i}$ 10. for $\mathrm{j} \leftarrow 1$ to $\mathrm{m}_{\mathrm{i}}$

11. Send 3-tuple $\left(\boldsymbol{T C H} \boldsymbol{H}_{i}, \boldsymbol{T D M A} \boldsymbol{A}_{i}, \boldsymbol{d}_{\text {mean }} \boldsymbol{i}^{j}\right)$ data to node $_{j}$

12. End For

13. End For

/* End of Setup Phase */

14 . For $\mathrm{v} \leftarrow 1$ to round

$/$ round $\rightarrow$ Total no. of rounds
} 


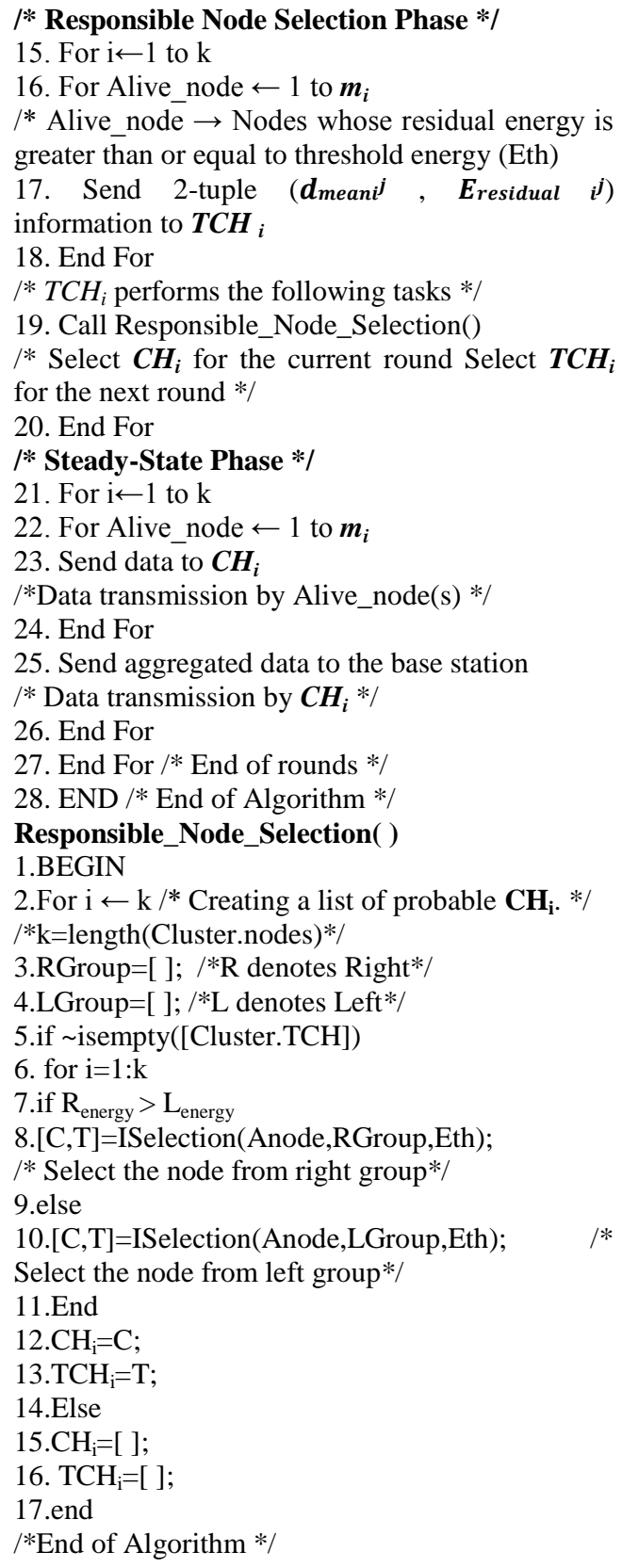

\section{PERFORMANCE COMPARISON}

\subsection{Qualitative Analysis}

The network lifetime is defined in terms of energy-usage. The associate list is determined in such a manner that presence of cluster head within a list is sufficient and further, to keep the network alive, probable list needs to be associated. To declare the network alive, we can say that for every round the cluster head with maximum energy among all the pre-decided cluster heads within that list should be greater than a threshold energy level $\mathrm{E}_{\text {threshold }}$ beyond which the node is considered as a dead. Since this must hold for every round, we can easily considered that only the minimum of these energies should be above $\mathrm{E}_{\text {threshold }}$ thereby guarantees that each round has sufficient energy to keep the network alive.

\subsection{Quantitative Analysis}

\subsubsection{Simulation Environment:}

To evaluate the performance of EEACPSC, MATLAB 7.1 is used as a simulation tool. We consider that the sensor nodes are deployed randomly across in plain area of $\left(\mathrm{x}_{\mathrm{m}}=100 \mathrm{~m} X\right.$ $\left.\mathrm{y}_{\mathrm{m}}=100 \mathrm{~m}\right)$ and the base station is located $(\mathrm{x}=50, \mathrm{y}=175)$. Each node is equipped with equal amount of initial energy (2J) at the beginning of the simulation. Further we assume that WSN is working in continuous data flow application domain. Table 1 represents various parameters and their values used in simulation.

TABLE 1. PARAMETERS AND CORRESPONDING VALUES USED IN SIMULATION

\begin{tabular}{|c|c|}
\hline Parameter & Parameter's Name \\
\hline Network Area & $100 \mathrm{~m} \mathrm{X} 100 \mathrm{~m}$ \\
\hline Base Station's Position & $(50 \mathrm{~m}, 175 \mathrm{~m})$ \\
\hline Initial Energy for Nodes & $2 \mathrm{Joule}$ \\
\hline Number of Deployed Nodes & 100 \\
\hline Size of Data Message & $4000 \mathrm{bits}$ \\
\hline $\begin{array}{c}\text { Energy Consumed in Data } \\
\left.\text { Aggregation( } \mathrm{E}_{\mathrm{DA}}\right)\end{array}$ & $5 \mathrm{~nJ} / \mathrm{bit} / \mathrm{signal}$ \\
\hline $\begin{array}{c}\text { Energy Consumed by } \\
\left.\text { Transceiver's Circuitry( } \mathrm{E}_{\mathrm{elec}}\right)\end{array}$ & $50 \mathrm{~nJ} / \mathrm{bit}$ \\
\hline $\begin{array}{c}\text { Energy Expenditure in Transmit- } \\
\text { Amplification in free-space } \\
\left.\text { model( } \mathrm{e}_{\mathrm{fs}}\right)\end{array}$ & $10 \mathrm{pJ} / \mathrm{bit} / \mathrm{m}^{2}$ \\
\hline $\begin{array}{c}\text { Energy Expenditure Transmit- } \\
\text { Amplification in multipath fading } \\
\text { model }\left(\mathrm{E}_{\mathrm{mp}}\right)\end{array}$ & $0.0013 \mathrm{pJ} / \mathrm{bit} / \mathrm{m}^{4}$ \\
\hline
\end{tabular}

\subsubsection{Simulation Metric:}

The performance of the scheme is evaluated considering network lifetime as a parameter which is defined as the time until the last node dies in the network. Network lifetime is measured using following yard-stick.

- Number of nodes alive in the network $\rightarrow$ more number of nodes alive involves network lifetime last longer.

- $3 \mathrm{D}$ result $\rightarrow$ results are in 3D format which outperforms better performance as compare to E3PSC and EACPLEP.

\subsubsection{Result and Discussion:}

A set of experiments is conducted to compare the performance of present scheme EEACPSC with E3PSC [7] and EACPLEP [8].

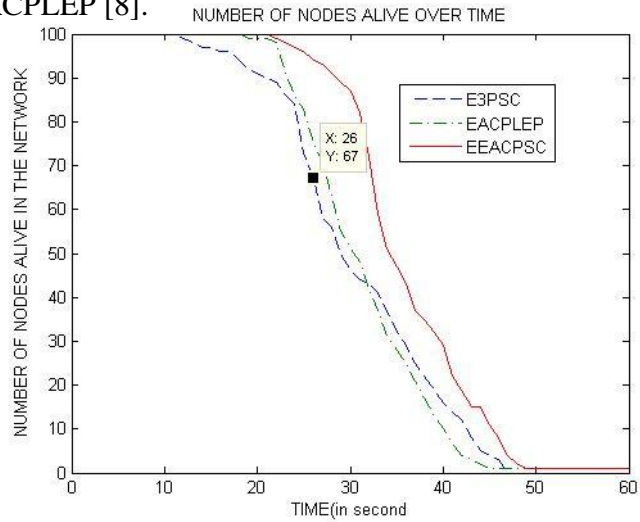

Fig. 1. Comparison of EEACPSC with E3PSC in terms of number of nodes alive in the network over time

Fig. 1 shows that, after 26 seconds, only 67 nodes are alive over the network. 


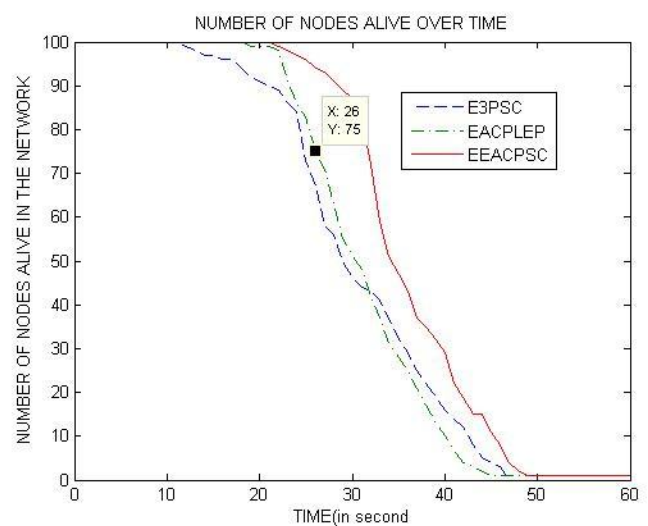

Fig. 2. Comparison of EEACPSC with EACPLEP in terms of number of nodes alive in the network over time

Fig. 2 shows that, after 26 seconds, only 75 nodes are alive over the network.

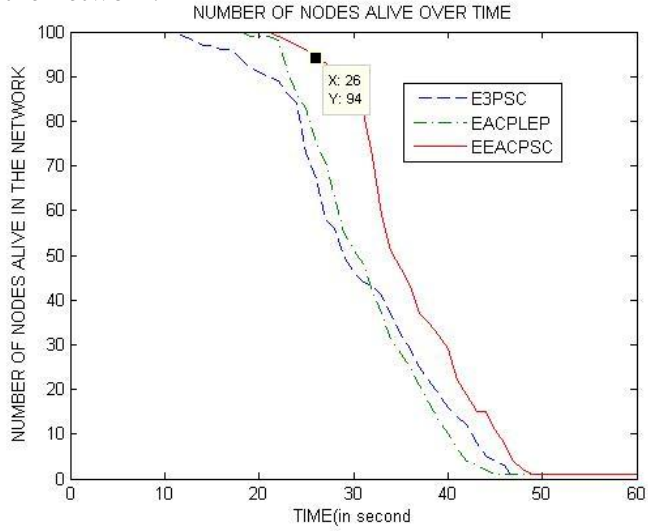

Fig.3. Number of nodes alive in the network over time in EEACPSC

Fig. 3 shows that, after 26 seconds, 94 nodes are alive over the network.

After comparing these 3 graphs we conclude that the performance of present scheme is much better than the previous one. And it provides better result. $40.3 \%$ gain of EEACPSC over E3PSC and $25.3 \%$ gain over EACPLEP in terms of number of nodes alive in the network. Following are the representation of 3D graphs.

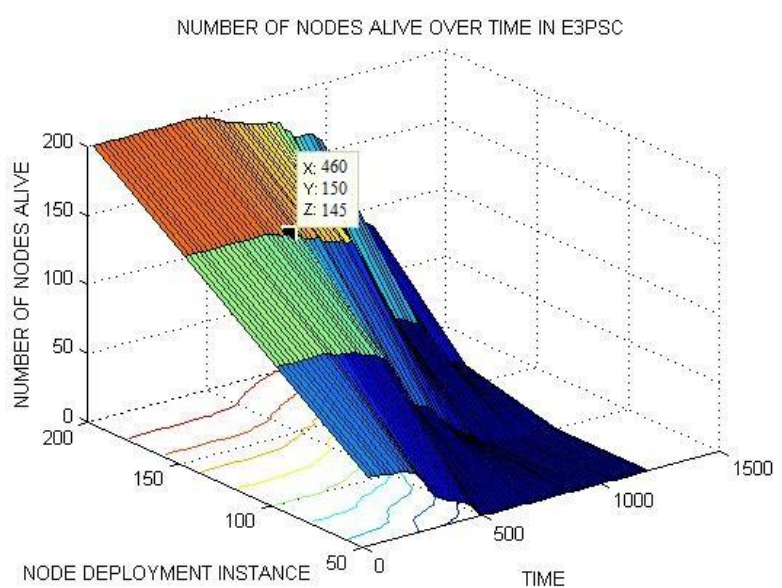

Fig4. Number of nodes alive over time in E3PSC (3D)
In fig 4, there is a deployment of 150 nodes instantly and after 460 seconds there are 145 nodes alive in the network. NUMBER OF NODES ALIVE OVER TIME IN EACPLEP

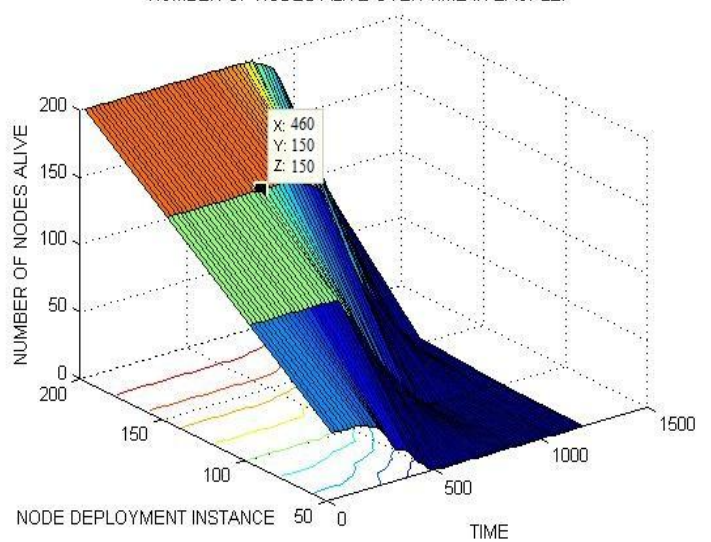

Fig. 5. Number of nodes alive over time in EACPLEP (3D)

Fig. 5 indicates that, after 460 seconds, there are 150 nodes alive over the network.

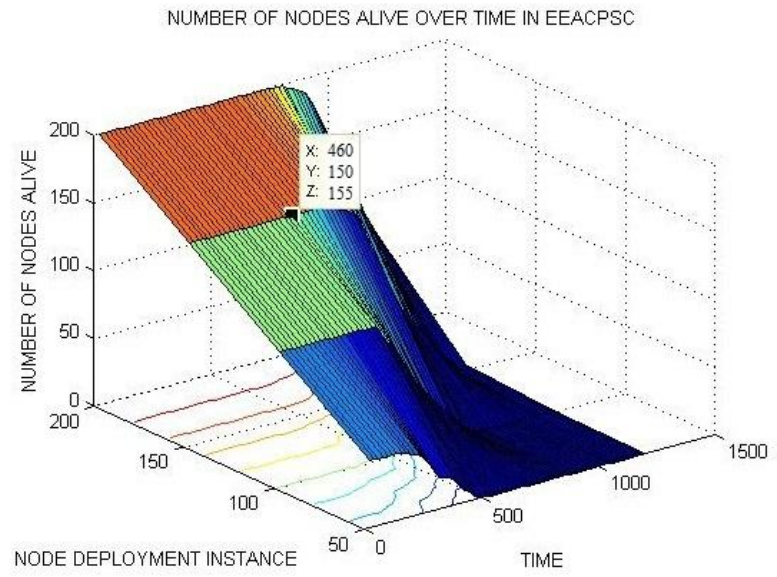

Fig. 6. Number of nodes alive over time in EEACPSC(3D)

Fig. 6 shows that, after 460 seconds, 155 nodes are alive over the network.

After comparing these 3D graphs, we conclude that the performance of present scheme is better as compare to previous scheme. $6.89 \%$ gain of EEACPSC over E3PSC and $3.33 \%$ gain over EACPLEP in terms of number of nodes alive in the network.

\section{CONCLUSION}

In this paper an energy-efficient routing scheme EEACPSC for WSN is proposed. The EEACPSC is developed by modifying an existing routing scheme E3PSC and EACPLEP with an objective to prolong network lifetime further (more number of nodes alive in the network). With the results of simulation, we can conclude that in the case of EACPLEP, the intra-cluster communication is increased ,which is removed in the EEACPSC, because the cluster head will be choose on the basis of creating a probable list of nodes. So the energy dissipated in the node to cluster head is less than the EACPLEP. And it organizes by the 3D graph also. So we can say that EEACPSC is more energy efficient and coverage preserving as compare to E3PSC and EACPLEP and spatial allocation of nodes plays a vital role in the election of cluster-heads for network operation. As per the quantitative results, EEACPSC proves to be better performer than EACPLEP and E3PSC. 


\section{FUTURE WORK}

As future work, we intend to study more complicated situations, like coverage problems in case of mobile nodes, heterogeneous sensor networks consisting nodes of different capabilities, and other non-disk coverage models.

\section{REFERENCES}

[1] D. J. Cook and S. K. Das, "Smart environments: technologies, protocols and applications," New York: John Wiley, pp. 13-15, 2004.

[2] K. Sohraby, D. Minoli, and T. Znati, "Wireless sensor networks: technology, protocols and applications," New Jersey: John Wiley, pp. 38-71, 2007.

[3] I. F. Akyildiz, W. Su, Y. Sankarasubramaniam, and E. Cayirci, "Wireless sensor networks: A survey," Computer Networks, vol. 38, pp. 393-422, 2002.

[4] W. R. Heinzelman, A. P. Chandrakasan, and H. Balakrishnan, "Energy- Efficient Communication Protocol for Wireless Microsensor Networks," Proc. 33rd Hawaii Int'l. Conf. Sys. Sci., Jan. 2000.
[5] S. Lindsey, C. Raghavendra, and K. M. Sivalingam, "Data Gathering Algorithms in Sensor Networks using Energy Metrics," IEEE Trans. Parallel and Distrib. Sys., vol. 13, no. 9, Sept. 2002, pp. 924-35.

[6] Amir Sepasi Zahmati and Bahman Abolhassani, "EPMPLCS: An Efficient Power Management Protocol with Limited Cluster Size for Wireless Sensor Networks", Proc. 27th International Conference on Distributed Computing Systems (ICDCS 2007), submitted for publication.

[7] S.K. Chaurasiya, Tumpa Pal, and Sipra Das Bit, "An Enhanced Energy-Efficient Protocol with static Clustering for WSN ", IEEE,Vol. 3, No. 11,2011, pp. 658-663.

[8] Arti Rana, Anshika Garg and Vivek Kumar, "Energy Aware Coverage Preservation and Lifetime Enhancement Protocol for WSN", in IJCA (0975-8887) Volume 100No. 11, Aug. 2014 